\title{
Higher baseline blood glucose is associated with reduced likelihood for successful recanalization in patients with basilar artery occlusion
}

\author{
Gabriel Broocks ${ }^{1} \oplus$ - Maximilian Groffmann ${ }^{1} \cdot$ Lukas Meyer $^{1} \cdot$ Sarah Elsayed ${ }^{1} \cdot$ Helge Kniep $^{1} \cdot$ Andre Kemmling $^{2,3}$. \\ Noel van Horn ${ }^{1} \cdot$ Rosalie McDonough $^{1} \cdot$ Tobias D. Faizy $^{1} \cdot$ Matthias Bechstein $^{1} \cdot$ Peter Sporns ${ }^{4}$. Thilo Rusche ${ }^{4,5}$. \\ Gerhard Schön $^{6} \cdot$ Jawed Nawabi $^{7,8} \cdot$ Jens Fiehler ${ }^{1} \cdot$ Uta Hanning $^{1}$
}

Received: 16 November 2021 / Revised: 22 December 2021 / Accepted: 23 December 2021 / Published online: 4 January 2022

(c) The Author(s) 2022

\begin{abstract}
Purpose Evidence regarding the effect of mechanical thrombectomy (MT) of basilar artery occlusion (BAO) stroke is yet sparse. As successful recanalization has been suggested as major determinant of outcome, the early identification of modifiable factors associated with successful recanalization could be of importance to improve functional outcome. Hyperglycemia has been associated with enhanced thrombin generation and unfavorably altered clot features.

Objective We hypothesized that serum baseline glucose is associated with likelihood of vessel recanalization mediated by collateral quality and clot burden in BAO stroke.

Methods BAO stroke patients who received multimodal CT on admission were analyzed. The association of vessel recanalization defined using modified Thrombolysis in cerebral infarction scale (mTICI) scores $2 b-3$, and baseline imaging and clinical parameters were tested in logistic regression analyses. Collateral quality and clot burden were evaluated using the Basilar Artery on CT-Angiography (BATMAN) score.

Results Out of 117 BAO patients, 91 patients (78\%) underwent MT. In 70 patients (77\%), successful recanalization could be achieved (mTICI $2 \mathrm{~b} / 3$ ). In multivariable logistic regression analysis, only a higher BGL (aOR 0.97, 95\% CI 0.96-0.99, $p=0.03$ ) and higher BATMAN score (aOR 1.77, 95\% CI 1.11-2.82, $p=0.02$ ) were independently associated with vessel recanalization. Application of alteplase, or time from symptom onset-imaging revealed no independent association with recanalization status.

Conclusion Higher BGL was significantly associated with reduced likelihood for recanalization success besides BATMAN score as a measure of collateral quality and clot burden. BGL could be tested as a modifiable parameter to increase likelihood for recanalization in BAO stroke, aiming to improve functional outcome.
\end{abstract}

Keywords Stroke $\cdot$ Ischemia $\cdot$ Infarction · Thrombolysis · Computed Tomography

\section{Abbreviations}

CT Computed tomography

MT Mechanical thrombectomy
BATMAN

NIHSS

BAO
Basilar artery on CT-angiography National Institute of Health Stroke Scale Basilar artery occlusion
Gabriel Broocks

g.broocks@uke.de

1 Department of Diagnostic and Interventional Neuroradiology, University Medical Center HamburgEppendorf, Martinistrasse 52, 20246 Hamburg, Germany

2 Department of Neuroradiology, Westpfalz-Klinikum, Kaiserslautern, Germany

3 Department of Neuroradiology, University Hospital Marburg, Marburg, Germany

4 Department of Neuroradiology, Universitätsspital Basel, Basel, Switzerland
5 Department of Radiology, University Hospital Münster, Münster, Germany

6 Institute of Medical Biometry and Epidemiology, University Medical Center Hamburg-Eppendorf, Hamburg, Germany

7 Department of Radiology, Charité University Medical Center, Berlin, Germany

8 Berlin Institute of Health (BIH), BIH Biomedical Innovation Academy, Berlin, Germany 


$\begin{array}{ll}\text { NECT } & \begin{array}{l}\text { Non-enhanced computed tomography } \\ \text { Modified Thrombolysis in cerebral infarc- } \\ \text { tion scale }\end{array} \\ \text { BGL } & \begin{array}{l}\text { Baseline serum glucose levels } \\ \text { pcASPECTS }\end{array} \\ & \begin{array}{l}\text { Posterior circulation Acute Stroke Progno- } \\ \text { sis Early CT score }\end{array}\end{array}$

\section{Introduction}

Mechanical thrombectomy (MT) is associated with improved functional outcome when performed in patients with ischemic stroke and large vessel occlusion in the anterior circulation [11, 20, 27]. For patients presenting with an acute occlusion of the basilar artery (BAO), however, evidence is yet spare, although this patient population represents about $20 \%$ of all stroke patients [12]. Successful vessel recanalization has been described as the most important predictor of good functional outcome in BAO stroke [7]. Accordingly, failed endovascular recanalization was associated with a 13-fold increase of risk for poor outcome. Hence, the early identification of factors, especially modifiable factors, associated with successful recanalization could be of clinical importance to improve functional outcome in BAO patients.

In patients with acute coronary syndrome, prior studies observed that elevated glucose levels are associated with enhanced thrombin formation, platelet activation, and fibrin clot resistance [28]. For patients with ischemic stroke, it has been described that hyperglycemia may increase infarct volume, risk for secondary hemorrhage, and finally lead to a worse prognosis $[17,18]$. Furthermore, observations in alteplase-treated patients confirmed that acute hyperglycemia may impede the fibrinolytic process potentially affecting reperfusion. Hence, it has been concluded that early measures to reduce elevated blood glucose levels could improve early recanalization [19]. Yet, the impact of baseline serum glucose levels (BGL) on recanalization success, especially in BAO stroke patients, remains uncertain. While acknowledging a history of diabetes mellitus as potential factor, most previous studies investigating reasons for unsuccessful recanalization did not specifically analyze BGL $[10,13]$. A recent study observed that diabetes mellitus was associated with an increased likelihood of a first pass effect [14]. That raises the question, whether patients with known diabetes treated adequately could exhibit lower glucose levels on admission, compared to (often elderly) patients with prediabetes or undiagnosed diabetes and subsequent higher baseline BGL. Anti-diabetic treatment might also have an impact on the likelihood of reperfusion, and hence better functional outcome. In line with this, a previous study observed that stroke patients medicated with sulfonylureas had better outcomes [16]. Yet, it has not been investigated whether BGL is associated with likelihood of recanalization. Better knowledge of the role of BGL in BAO stroke may be of high clinical relevance, considering that in contrast to anterior circulation stroke, diagnosis is often delayed and reperfusion rates are very low in the absence of MT (i.e. $4 \%[2])[7,8]$.

The purpose of this study was to investigate factors associated with successful recanalization in BAO stroke, in particular BGL. As the status of intracranial collaterals might mediate the effect of BGL, we also sought to investigate the relationship of BGL and collateral quality and its combined diagnostic accuracy to predict vessel recanalization. We hypothesized that BGL are associated with likelihood of recanalization.

\section{Methods}

\section{Patient cohort}

All patients admitted with acute ischemic posterior circulation stroke between June 2015 and February 2019 at two high-volume tertiary stroke centers were retrospectively analyzed. Anonymized data were recorded and analyzed in accordance with ethical guidelines and after approval of the local ethics committee (IRB approval number WF04/13). Informed consent was waived.

The inclusion criteria for this study were: (1) acute ischemic posterior circulation stroke with multimodal CT imaging on admission [non-enhanced CT (NECT), CTangiography (CTA) and CT perfusion (CTP)]; (2) occlusion of the basilar artery apparent on CTA; (3) last known well $<24 \mathrm{~h}$; (4) absence of intracranial hemorrhage; (5) absence of significant imaging artifacts.

Successful endovascular recanalization was defined by modified Thrombolysis in Cerebral Infarction (mTICI)-classification as mTICI score $\geq 2 \mathrm{~b}$ at the conclusion of the procedure by the performing neurointerventionalist, validated by a further attending neuroradiologist.

\section{Image analysis}

To assess the degree of early ischemic changes, Posterior circulation Acute Stroke Prognosis Early CT score (pcASPECTS) was rated by two experienced board-certified neuroradiologists, and discrepancies were settled by joint discussion of both readers. The collateral quality and clot burden was evaluated using the Basilar Artery on CT-Angiography (BATMAN) score, a semiquantitative CTA-based grading system [1]. Consequently, the vertebrobasilar system was divided into six segments [vertebral arteries $=1$, basilar artery $=2-4$, posterior cerebral artery, $\mathrm{PCA}=5$, posterior communicating artery $(\mathrm{Pcom})=6]$. Points were allocated, as 
described: 2 points for each Pcom, 1 point for a hypoplastic Pcom $(<1 \mathrm{~mm})$ if in continuity with the basilar artery top via P1-posterior cerebral artery (PCA), or 3 points for each fetal Pcom, 1 point for each of the other segments (= maximum score of 10). Rating of BATMAN was performed by two experienced board-certified neuroradiologists who were blinded to the scores of the other reader. Discrepancies about the BATMAN score were settled by joint discussion of the two readers.

\section{Image acquisitions}

All included patients received a comprehensive stroke imaging protocol at admission with NECT and CTA and additional CTP consecutively in equal order on an iCT $256^{\mathrm{TM}}$ scanner (Philips Healthcare, Best, The Netherlands). NECT: collimation $64 \times 0.625$, pitch 0.297 , rotation time $0.4 \mathrm{~s}$, FOV $270 \mathrm{~mm}$, tube voltage $120 \mathrm{kV}$, tube current $300 \mathrm{~mA}$, $4.0 \mathrm{~mm}$ slice reconstruction. CTA: collimation $64 \times 0.625$, pitch 0.985 , rotation time $0.4 \mathrm{~s}$, FOV $220 \mathrm{~mm}$, tube voltage $120 \mathrm{kV}, 300 \mathrm{mAs}, 2.0 \mathrm{~mm}$ slice reconstruction, $5 \mathrm{~mm}$ MIP reconstruction with $1 \mathrm{~mm}$ increment. CTP: collimation $64 \times 1.25$, rotation time $0.5 \mathrm{~s}$, FOV $220 \mathrm{~mm}$, tube current $80 \mathrm{kV}$, tube current $140 \mathrm{mAs}, 5 \mathrm{~mm}$ slice reconstruction, slice sampling rate $1.8 \mathrm{~s}$, scan time $72 \mathrm{~s}$, biphasic injection with $40 \mathrm{ml}$ of highly iodinated contrast medium with $400 \mathrm{mM} / \mathrm{ml}$ injected with $6 \mathrm{ml} / \mathrm{s}$ followed by $40 \mathrm{ml} \mathrm{NaCl}$ chaser bolus.

\section{Statistical analysis}

Categorial variables were compared using $\chi^{2}$ tests. Distribution of metric variables was described by means and standard deviation (SD) or medians and interquartile ranges (IQR). Kolmogorov-smirnov tests were used to test for normal distribution. Student $t$ tests (normal distribution) with SD or Mann-Whitney- $U$ tests (non-normal distribution) with IQR were used to determine differences of the acquired parameters for patients with versus without recanalization.

We defined a binary outcome (successful recanalization) as $\mathrm{mTICI}$ score $\geq 2 \mathrm{~b}$. Logistic regression analysis was performed to assess the association between the clinical and radiological parameters, and outcome (successful recanalization versus no successful recanalization). For univariable logistic regression analysis, all available independent variables (BGL, BATMAN score, time from onset to imaging, age, sex, application of alteplase, NIHSS on admission, and pcASPECTS) were analyzed with successful recanalization as dependent variable (Table 2). Second, multivariable logistic regression models were performed implementing the independent variables that showed a significant association in univariable analysis. Correlation between all independent variables was tested to exclude multicollinearity. The area under the curve for the resulting logistic model was calculated to show its diagnostic ability. The probability for vessel recanalization according to the BATMAN score was plotted separately for patients with different levels of BGL (trichotomized based on $25 \%$ and $75 \%$ percentile). Finally, it was analyzed whether the aforementioned variables and recanalization status are associated with functional outcome assessed using uni- and multivariable ordinal regression analysis with modified Rankin Scale (mRS) scores at day 90 as dependent variable.

A statistically significant difference was accepted at a $p$ value of less than 0.05 . Analyses were performed using MedCalc (version 11.5.1.0; Mariakerke, Belgium) and Stata 13.0 (StataSE, StataCorp, TX, USA).

\section{Results}

\section{Patients}

The patient characteristics including baseline, treatment, and outcome variables are displayed in Table 1, separately for patients with successful recanalization versus patients without successful recanalization. Figure 1 shows an example of a patient with high BGL and low BATMAN score. A patient inclusion flow chart is included in the supplemental material. A total of 117 patients were included. The mean age was 69 years (SD 13.6) and 46 patients were female (39\%). In 82 patients (70\%), time from symptom onset to imaging was known. In these patients, the median time from symptom onset to admission imaging was $3.3 \mathrm{~h}$ (IQR 1.3-7.2 h). 35 patients (29\%) had an unknown onset (within $24 \mathrm{~h}$ from last known well). In total, 91 patients underwent MT (77\%), of which $70(77 \%)$ resulted in successful recanalization (mTICI 2b/3). Among 35 patients with unknown onset, 30 patients underwent MT (86\%), and 60 patients (73\%) from patients with known time window underwent MT $(p=0.14)$. The median NIHSS on admission was 20 (IQR 8-42). The median pcASPECTS was 9 (IQR 8-9) and the median BATMAN score was 7 (IQR 5-9). The median BGL was $134 \mathrm{mg} /$ dl (IQR 115-160). In 23 patients (20\%) a known history of diabetes mellitus type 2 was deducted from medical documentation. The median $\mathrm{mRS}$ score at day 90 was 4 (IQR 1-5). Table 1 displays the patient characteristics.

Comparing patients with successful recanalization to patients without, there was no significant difference in age, sex, or NIHSS on admission ( $p=0.21 / 0.41 / 0.09)$. Likewise, the rate of patients with unknown onset within $24 \mathrm{~h}$ from last known well was not different, although patients with successful recanalization offered a shorter time from known onset of stroke symptoms ( $2.5 \mathrm{~h}$ versus $4.0 \mathrm{~h}, p=0.04$ ). Moreover, patients with successful recanalization had a 
Table 1 Patient characteristics

\begin{tabular}{llll}
\hline & $\begin{array}{l}\text { MT with mTICI 2b-3 } \\
n=70\end{array}$ & $\begin{array}{l}\text { No MT/MT with } \\
\text { mTICI 0-2a } \\
n=47\end{array}$ & $p$ value \\
\hline Baseline characteristics & & & \\
Age in years, mean (SD) & $68(1.8)$ & $71(1.5)$ & 0.21 \\
Sex female, $n$ (\%) & $25(36)$ & $21(44)$ & 0.41 \\
Known time from onset to imaging in & $2.5(1.2-6.0)$ & $4.0(2.0-12.0)$ & 0.04 \\
$\quad$ hours, median (IQR) & $24(35)$ & $11(23)$ & 0.17 \\
Unknown onset within 24 h, $n(\%)$ & $22(12-42)$ & $18(4-36)$ & 0.09 \\
NIHSS on admission, median (IQR) & $126(111-149)$ & $144(120-177)$ & 0.01 \\
Glucose, median mg/dl (IQR) & $14(20)$ & $9(18)$ & 0.21 \\
History of Diabetes, $n$ (\%) & $7(6-9)$ & $5(5-7)$ & 0.02 \\
BATMAN score, median (IQR) & $9(8-10)$ & $9(8-9)$ & 0.07 \\
pcASPECTS, median (IQR) & & & \\
Treatment and follow-up & $38(55)$ & $17(29)$ & 0.04 \\
IV alteplase, $n$ (\%) & $3(1-15)$ & $5(1-6)$ & 0.09 \\
NIHSS at discharge, median (IQR) & $4(1-5)$ & 0.19 \\
mRS after 90 days, median (IQR) & $4.23)$ & \\
\hline
\end{tabular}

$I Q R$ indicates interquartile range, NIHSS National Institute of Health Stroke Scale, pcASPECTS posterior circulation Alberta Stroke Program Early CT Score, IV intravenous, TICI thrombolysis in cerebral infarctions, $m R S$ modified Ranking Scale

Table 2 Binary logistic regression analysis for successful recanalization (TICI $2 b / 3)$

\begin{tabular}{|c|c|c|c|c|c|c|}
\hline & \multicolumn{3}{|c|}{ Univariable analysis $^{\mathrm{a}}$} & \multicolumn{3}{|c|}{ Multivariable analysis } \\
\hline & OR & $95 \% \mathrm{CI}$ & $p$ value & OR & $95 \% \mathrm{CI}$ & $p$ value \\
\hline Time onset-imaging & 0.92 & $0.86-0.99$ & 0.03 & 0.89 & $0.75-1.06$ & $>0.4$ \\
\hline BGL & 0.98 & $0.98-0.99$ & 0.01 & 0.98 & $0.95-0.99$ & 0.03 \\
\hline BATMAN & 1.51 & $1.07-2.13$ & 0.02 & 1.87 & $1.07-3.29$ & 0.03 \\
\hline IV alteplase & 2.24 & $1.05-4.78$ & 0.04 & 1.12 & $0.13-4.79$ & $>0.3$ \\
\hline Age & 0.98 & $0.95-1.01$ & 0.21 & - & - & - \\
\hline Sex & 0.73 & $0.34-1.55$ & 0.41 & - & - & - \\
\hline NIHSS & 1.02 & $0.99-1.04$ & 0.18 & - & - & - \\
\hline pcASPECTS & 1.19 & $0.94-1.50$ & 0.14 & - & - & - \\
\hline
\end{tabular}

${ }^{\mathrm{a}}$ Age, Sex, NIHSS on admission, and pcASPECTS were not significantly associated with recanalization in univariable analysis significantly higher BATMAN score (7 versus $5, p=0.02$ ), lower BGL (126 mg/dl versus $144 \mathrm{mg} / \mathrm{dl}, p=0.01)$, and received intravenous alteplase more frequently (55\% versus $29 \%, p=0.04)($ Table 1$)$.

\section{Prediction of successful recanalization}

For this analysis, only patients undergoing MT $(n=91)$ were included. There was no significant correlation between the independent variables $(r< \pm 0.2)$. In univariable logistic regression analysis, BGL, BATMAN score, intravenous alteplase, and time from onset to imaging were significantly associated with successful recanalization (Table 2). No association between age, sex, pcASPECTS, or NIHSS and recanalization was observed.
In multivariable logistic regression analysis, BGL (OR $0.98,95 \%$ CI 0.95-0.99, $p=0.03$ ) and BATMAN (OR 1.87, $95 \%$ CI $1.07-3.29, p=0.03$ ) remained the only variables independently associated with successful recanalization (Fig. 2, Table 2). The diagnostic ability to classify recanalization status of this predictive model based on the area under the ROC curve was excellent (AUC 0.91). Figure 3 shows the incremental change of likelihood for recanalization based on the BATMAN score separately for patients with low $(<25 \%$ percentile, $<136 \mathrm{mg} / \mathrm{dl})$ intermediate (25-75\% percentile, $136-160 \mathrm{mg} / \mathrm{dl}$, and high BGL ( $>75 \%$ percentile, $>160 \mathrm{mg} / \mathrm{dl})$. 
Fig. 1 Example of a patient with low BATMAN score and high BGL. Illustration of a patient with acute basilar artery occlusion, poor BATMAN score, and high BGL on admission

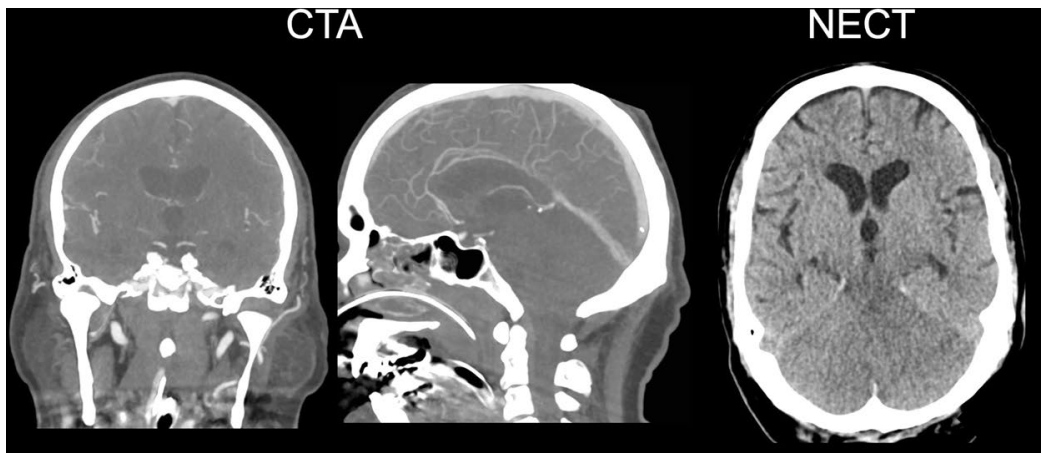

Acute BAO, 4.5 hours from onset, BGL: $198 \mathrm{mg} / \mathrm{dl}$, Very poor outcome (mRS 6) BATMAN score: 4 pcASPECTS: 3

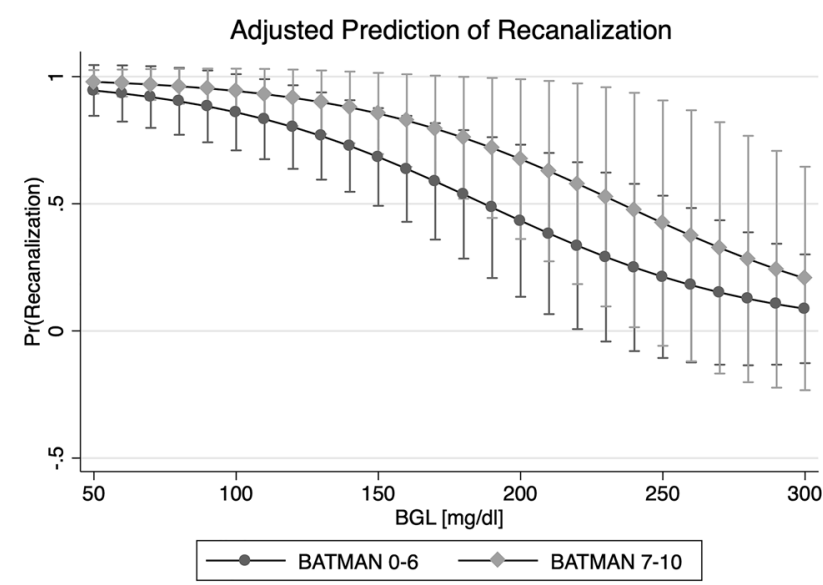

Fig. 2 Association of baseline glucose level and dichotomized BATMAN score on the likelihood for recanalization. Multivariable logistic regression plot to illustrate the impact of glucose ( $x$ axis, in $\mathrm{mg} / \mathrm{dl}$ ) and BATMAN score (dichotomized) on the likelihood of successful recanalization

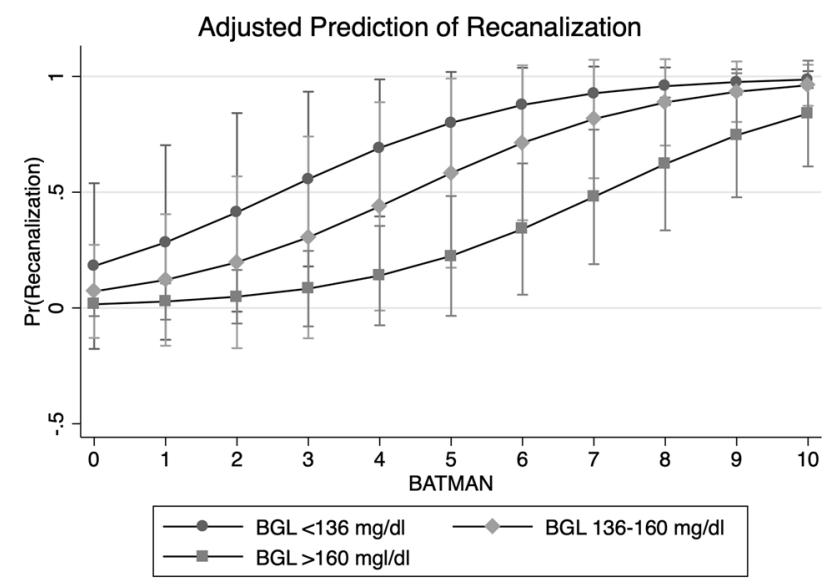

Fig. 3 Association of BATMAN score and trichotomized baseline glucose level on likelihood for recanalization. Multivariable logistic regression plot to illustrate the impact of the BATMAN score ( $x$ axis) and trichotomized blood glucose level (BGL) on the likelihood of successful recanalization

\section{Prediction of functional outcome}

Ordinal regression analysis was performed to identify variables associated with functional outcome at day 90. In univariable ordinal regression analysis, higher BGL was by trend associated with a higher $\mathrm{mRS}$ at day 90 (OR 1.007, 95\% CI $0.999-1.013, p=0.06)$. Successful recanalization was not associated with functional outcome $(p>0.1)$. After adding BATMAN, and BGL to the model, however, successful recanalization was significantly associated with functional outcome (OR for mRS shift: $0.18,95 \%$ CI $0.04-0.78, p=0.02$ ). In multivariable ordinal regression analysis, vessel recanalization, pcASPECTS, and NIHSS were independently associated with clinical outcome, adjusted for BATMAN, BGL, intravenous alteplase, and age (Table 3) (Fig. 4).

\section{Discussion}

The aim of this study was to investigate factors associated with successful recanalization in acute BAO patients, and in particular, analyze how baseline BGL and the BATMAN score, as a measure of collateral quality and clot burden, are associated with reperfusion. We hypothesized that BGL is directly related to the likelihood of successful recanalization, mediated by the BATMAN score. The study has several findings: (1) in multivariable logistic regression analysis, only BGL and BATMAN were independently associated with recanalization; (2) successful vessel recanalization was significantly associated with better functional outcome when adjusted for BGL and BATMAN score; (3) pcASPECTS and recanalization status were important predictors of functional outcome, while other variables such as application of intravenous alteplase, or time from onset to imaging, only had a minor impact on recanalization, and functional outcome.

In ischemic posterior circulation stroke, BAO is still associated with very poor functional outcome, despite recent 
Table 3 Uni- and multivariable ordinal regression analysis for clinical outcome (mRS shift)

\begin{tabular}{|c|c|c|c|c|c|c|}
\hline & \multicolumn{3}{|c|}{ Univariable analysis } & \multicolumn{3}{|c|}{ Multivariable analysis } \\
\hline & OR & $95 \% \mathrm{CI}$ & $p$ value & OR & $95 \% \mathrm{CI}$ & $p$ value \\
\hline BGL & 1.00 & $0.99-1.01$ & 0.06 & 1.01 & $0.99-1.02$ & $>0.3$ \\
\hline BATMAN & 0.84 & $0.66-1.08$ & 0.17 & 1.14 & $0.86-1.52$ & $>0.3$ \\
\hline Recanalization & 0.65 & $0.34-1.25$ & 0.20 & 0.18 & $0.04-0.84$ & 0.03 \\
\hline NIHSS & 1.08 & $1.06-1.11$ & $<0.001$ & 1.05 & $1.00-1.10$ & 0.04 \\
\hline IV alteplase & 1.48 & $0.78-2.82$ & 0.23 & 1.10 & $0.30-4.00$ & $>0.8$ \\
\hline pcASPECTS & 0.72 & $0.58-0.89$ & 0.002 & 0.58 & $0.39-0.86$ & $<0.01$ \\
\hline Age & 1.03 & $1.00-1.06$ & 0.01 & 1.04 & $0.38-0.86$ & 0.08 \\
\hline Time & 0.98 & $0.96-1.00$ & 0.14 & 1.07 & $0.92-1.24$ & $>0.5$ \\
\hline
\end{tabular}

$B G L$ baseline glucose level, Time Time from symptom onset to admission

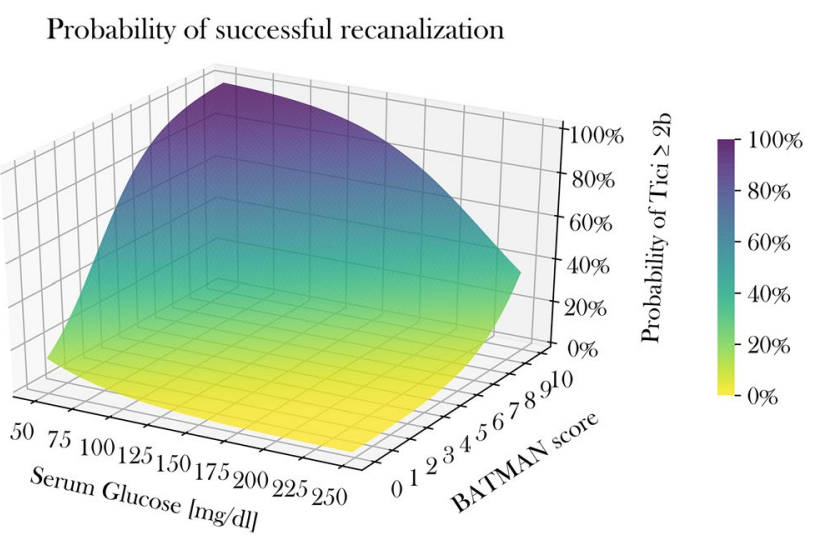

Fig. 4 3D Illustration of the relationship of glucose levels and BATMAN score on the likelihood for recanalization. 3D Surface plot to illustrate the impact of BATMAN scores and BGL on the likelihood for successful recanalization

advances in endovascular treatment. Lately, the results of the Basilar Artery International Cooperation Study (BASICS), analyzing the safety and efficacy of MT in BAO patients, have been presented (clinical trial ID: NCT01717755 [23]). This study observed no significant benefit of MT compared to best medical management alone, notwithstanding a slightly higher proportion of patients with good functional outcome. Despite controversies about the study design and particularly its long recruitment period, the study emphasizes a need to further improve treatment selection for MT and outcome prediction in BAO stroke.

Successful endovascular recanalization has been described as the most important predictor of good clinical outcome following BAO stroke, while failed thrombectomy was associated with a significant increase of risk for poor outcome [7, 9]. Therefore, the early identification of factors, in particular factors that can be adjusted in the acute situation, associated with successful recanalization might be of significant importance to improve functional outcome. In this study, only the BATMAN score, and BGL were independently associated with successful vessel recanalization.
While it has been observed that elevated BGL are associated with worse clinical outcome, it has not yet been described whether BGL may influence the likelihood of endovascular recanalization. A recently published study showed that higher glucose levels on admission are independent predictors of failure of early neurological improvement ("fENI") after successful thrombectomy [29]. Moreover, patients with anterior circulation stroke and higher glucose levels showed worse outcome, but the effect of hyperglycemia was exacerbated in patients with incomplete MT. That leads to the question, whether hyperglycemia could reduce likelihood for successful MT, and hence be associated with worse outcomes [15]. Previous studies investigating the impact of BGL on outcome in patients with acute coronary syndrome observed that elevated glucose levels are associated with enhanced thrombin formation, platelet activation, and fibrin clot [28]. In acute ischemic stroke, previous studies described that hyperglycemia may increase infarct volume, risk for secondary hemorrhage, and finally lead to a poor prognosis and that hyperglycemia could impede the fibrinolytic process potentially affecting reperfusion $[17,19]$. A further clinical trial evaluated the effects of hyperglycemia on recanalization rates and outcome in patients receiving intravenous alteplase, and the authors concluded that higher serum glucose was associated with lower rates of recanalization and worse outcome [22]. The question remained whether strict control of BGL might raise the likelihood for reperfusion. It is, however, important to realize that recanalization of BAO after intravenous alteplase alone occurs rarely. A prior study observed that only $4 \%$ of BAO patients may show recanalization after alteplase alone [2]. Therefore, the aforementioned observations on patients receiving alteplase-only cannot be directly related to patient cohorts undergoing thrombectomy. In contrast to BGL and BATMAN score, there was no independent association between the application of intravenous alteplase, or time from symptom onset to imaging and recanalization. Furthermore, it remains uncertain whether persistent higher BGL or acute BGL increases are causative and to what extent. Future 
research should include $\mathrm{HbA1c}$ and serial BGL measurements to elucidate this coherence.

The second prognostic factor that was associated with likelihood of recanalization was the BATMAN score, determined in baseline CTA. CT is the most widely performed imaging modality in acute stroke, and CTA the standard approach to diagnose $\mathrm{BAO}$, allowing the noninvasive assessment of the collateral status, which might have an important impact on the outcome of BAO patients. The BATMAN score takes into account both the extent of the occlusion (i.e. number of obstructed perforating arteries and therefore extent of ischemia), and the presence of Pcom arteries that are known to be associated with a better prognosis in BAO [1]. It has been described that the additional assessment of collateral quality to clot burden improves the diagnostic accuracy in BAO stroke [1].

To our knowledge, this is the first study that investigated baseline variables associated with successful recanalization in BAO, and the first study to analyze how BGL directly affects the likelihood for vessel recanalization, depending on the collateral status, and clot burden. Analyzing factors associated with successful recanalization in BAO has particular clinical relevance considering the very poor outcomes associated with incomplete or failed MT and the comparably lower effect of intravenous alteplase (i.e. recanalization rate approximately $4 \%$ in BAO compared to approximately $30 \%$ in middle cerebral artery occlusion) $[2,7,8]$.

In accordance with a previous study, we observed in multivariable analysis that successful vessel recanalization is independently associated with better functional outcome (see Table 3) [7]. Besides recanalization status, pcASPECTS was an important predictor of outcome, although not associated with recanalization status. Nevertheless, pcASPECTS should be taken into account in the assessment of posterior circulation stroke patients. Patients with a low pcASPECTS are at particular risk of developing malignant cerebellar edema and should be monitored closely [3, 21].

In the clinical context, the assessment of the BATMAN score and acknowledgement of BGL might help to estimate the likelihood of recanalization, or even consider insistent normalization of BGL to improve chances for successful thrombectomy. A desirable side effect of BGL adjustment might be the reduction of ischemic edema, as recently described [5], which might have a further positive effect on functional outcome. Currently, anti-diabetic drugs are tested as adjuvant treatment option in ischemic stroke, such as Glyburide, an inhibitor of the sulfonylurea receptor-1, and transient receptor potential melastatin 4 (SUR1-TRPM4). It has been reported that glyburide might lower formation of ischemic brain edema, subsequently decreasing infarct volume, reducing mortality, and improving outcomes [16, 24, 26]. Edema reduction could constitute an interesting therapeutic target, as space-occupying edema is regularly associated with devastating outcomes [4]. Despite several previous studies testing neuroprotectants and therapeutic BGL adjustment to improve outcome in stroke, the subjects included in these studies did mostly not achieve reperfusion, which could significantly limit the effects of adjuvant treatment options [6, 25].

The results of the present study advocate the further implementation of imaging tools in the assessment of posterior circulation stroke patients in daily clinical practice, such as pcASPECTS, and the BATMAN score, as easy and fast methods to gain more insights into pathophysiological processes, which could be helpful particularly in cases with uncertain time window and/or limited clinical assessability.

There are several limitations of this study. First, this was a retrospective study with a limited number of patients and risk of selection bias. Furthermore, hyperglycemia during ischemic stroke is a dynamic process, and single assessments of BGL might not be sufficient to capture the complexity. Further studies could assess multiple measurements of BGL and analyze the impact of $\mathrm{HbA} 1 \mathrm{c}$ values that were not available in the present study, because they are not part of the routine laboratory for acute ischemic stroke patients. Higher BGL on admission might be accompanied by further unfavorable metabolic epiphenomena, and cardiovascular risk factors, leading to a potential bias. Finally, information on previous medication was not available. Future research is needed to investigate the potential impact of antidiabetic medication.

\section{Conclusion}

In this study, lower BGL and the higher BATMAN scores were the only independent predictors of successful vessel recanalization in acute $\mathrm{BAO}$, which was associated with better functional outcome at day 90 . BGL might constitute a modifiable variable to increase likelihood for successful treatment, and the impact of BGL adjustments might be tested in further prospective studies.

Supplementary Information The online version contains supplementary material available at https://doi.org/10.1007/s00415-021-10948-1.

Funding Open Access funding enabled and organized by Projekt DEAL. This research received no specific grant from any funding agency in the public, commercial or not-for-profit sectors.

Data availability The data that support the findings of this study are available from the corresponding author upon reasonable request.

\section{Declarations}

Conflicts of interest J. Fiehler: research support from the German Ministry of Science and Education (BMBF), German Ministry of Econ- 
omy and Innovation (BMWi), German Research Foundation (DFG), European Union (EU), Hamburgische Investitions-/Förderbank (IFB), Medtronic, Microvention, Philips, Stryker; consultancy appointments; Acandis, Bayer, Boehringer Ingelheim, Cerenovus, Covidien, Evasc Neurovascular, MD Clinicals, Medtronic, Medina, Microvention, Penumbra, Route92, Stryker, Transverse Medical; stock holdings for Tegus. Other authors: No disclosures.

Ethical approval Anonymized data were recorded and analyzed in accordance with ethical guidelines and after approval of the local ethics committee. Informed consent was waived.

Open Access This article is licensed under a Creative Commons Attribution 4.0 International License, which permits use, sharing, adaptation, distribution and reproduction in any medium or format, as long as you give appropriate credit to the original author(s) and the source, provide a link to the Creative Commons licence, and indicate if changes were made. The images or other third party material in this article are included in the article's Creative Commons licence, unless indicated otherwise in a credit line to the material. If material is not included in the article's Creative Commons licence and your intended use is not permitted by statutory regulation or exceeds the permitted use, you will need to obtain permission directly from the copyright holder. To view a copy of this licence, visit http://creativecommons.org/licenses/by/4.0/.

\section{References}

1. Alemseged F, Shah DG, Diomedi M, Sallustio F, Bivard A, Sharma G, Mitchell PJ, Dowling RJ, Bush S, Yan B, Caltagirone C, Floris R, Parsons MW, Levi CR, Davis SM, Campbell BC (2017) The basilar artery on computed tomography angiography prognostic score for basilar artery occlusion. Stroke 48:631-637

2. Bhatia R, Hill MD, Shobha N, Menon B, Bal S, Kochar P, Watson T, Goyal M, Demchuk AM (2010) Low rates of acute recanalization with intravenous recombinant tissue plasminogen activator in ischemic stroke: real-world experience and a call for action. Stroke 41:2254-2258

3. Broocks G, Elsayed S, Kniep H, Kemmling A, Flottmann F, Bechstein M, Faizy TD, Meyer L, Lindner T, Sporns P, Rusche T, Schon G, Mader MM, Nawabi J, Fiehler J, Hanning U (2021) Early prediction of malignant cerebellar edema in posterior circulation stroke using quantitative lesion water uptake. Neurosurgery 88:531-537

4. Broocks G, Elsayed S, Kniep H, Kemmling A, Flottmann F, Bechstein M, Faizy TD, Meyer L, Lindner T, Sporns P, Rusche T, Schon G, Mader MM, Nawabi J, Fiehler J, Hanning U (2020) Early prediction of malignant cerebellar edema in posterior circulation stroke using quantitative lesion water uptake. Neurosurgery

5. Broocks G, Kemmling A, Aberle J, Kniep H, Bechstein M, Flottmann F, Leischner H, Faizy TD, Nawabi J, Schon G, Sporns P, Thomalla G, Fiehler J, Hanning U (2020) Elevated blood glucose is associated with aggravated brain edema in acute stroke. J Neurol 267:440-448

6. Campbell BCV, Mitchell PJ, Churilov L, Yassi N, Kleinig TJ, Dowling RJ, Yan B, Bush SJ, Dewey HM, Thijs V, Scroop R, Simpson M, Brooks M, Asadi H, Wu TY, Shah DG, Wijeratne T, Ang T, Miteff F, Levi CR, Rodrigues E, Zhao H, Salvaris P, Garcia-Esperon C, Bailey P, Rice H, de Villiers L, Brown H, Redmond K, Leggett D, Fink JN, Collecutt W, Wong AA, Muller C, Coulthard A, Mitchell K, Clouston J, Mahady K, Field D, Ma H, Phan TG, Chong W, Chandra RV, Slater LA, Krause M, Harrington TJ, Faulder KC, Steinfort BS, Bladin CF, Sharma G, Desmond PM, Parsons MW, Donnan GA, Davis SM, Investigators
E-IT (2018) Tenecteplase versus alteplase before thrombectomy for ischemic stroke. N Engl J Med 378:1573-1582

7. Deb-Chatterji M, Flottmann F, Leischner H, Alegiani A, Brekenfeld C, Fiehler J, Gerloff C, Thomalla G (2020) Recanalization is the key for better outcome of thrombectomy in basilar artery occlusion. Clin Neuroradiol 30:769-775

8. Fabritius MP, Thierfelder KM, Meinel FG, Othman AE, Dorn F, Sabel BO, Scheffler P, Ertl-Wagner B, Sommer WH, Kunz WG (2017) Early Imaging prediction of malignant cerebellar edema development in acute ischemic stroke. Stroke 48:2597-2600

9. Flottmann F, Broocks G, Faizy TD, McDonough R, Watermann L, Deb-Chatterji M, Thomalla G, Herzberg M, Nolte CH, Fiehler J, Leischner H, Brekenfeld C, investigators GSR (2020) Factors associated with failure of reperfusion in endovascular therapy for acute ischemic stroke: a multicenter analysis. Clin Neuroradiol

10. Goda T, Oyama N, Kitano T, Iwamoto T, Yamashita S, Takai H, Matsubara S, Uno M, Yagita Y (2019) Factors associated with unsuccessful recanalization in mechanical thrombectomy for acute ischemic stroke. Cerebrovasc Dis Extra 9:107-113

11. Goyal M, Menon BK, van Zwam WH, Dippel DW, Mitchell PJ, Demchuk AM, Davalos A, Majoie CB, van der Lugt A, de Miquel MA, Donnan GA, Roos YB, Bonafe A, Jahan R, Diener HC, van den Berg LA, Levy EI, Berkhemer OA, Pereira VM, Rempel J, Millan M, Davis SM, Roy D, Thornton J, Roman LS, Ribo M, Beumer D, Stouch B, Brown S, Campbell BC, van Oostenbrugge RJ, Saver JL, Hill MD, Jovin TG, collaborators H (2016) Endovascular thrombectomy after large-vessel ischaemic stroke: a metaanalysis of individual patient data from five randomised trials. Lancet 387:1723-1731

12. Guenego A, Lucas L, Gory B, Richard S, Aubertin M, Weisenburger-Lile D, Labreuche J, Dargazanli C, Benali A, Bourcier R, Detraz L, Vannier S, Guillen M, Eugene F, Walker G, Lun R, Wormsbecker A, Ducroux C, Piotin M, Blanc R, Consoli A, Lapergue B, Fahed R, investigators E (2021) Thrombectomy for comatose patients with basilar artery occlusion: a multicenter study. Clin Neuroradiol

13. Heider DM, Simgen A, Wagenpfeil G, Dietrich P, Yilmaz U, Muhl-Benninghaus R, Roumia S, Fassbender K, Reith W, Kettner M (2020) Why we fail: mechanisms and co-factors of unsuccessful thrombectomy in acute ischemic stroke. Neurol Sci 41:1547-1555

14. Huo X, Liu R, Gao F, Ma N, Mo D, Liao X, Wang C, Sun X, Song L, Jia B, Liu L, Wang B, Pan Y, Wang Y, Liu L, Zhao X, Wang Y, Miao Z, Group ES (2019) Effect of hyperglycemia at presentation on outcomes in acute large artery occlusion patients treated with solitaire stent thrombectomy. Front Neurol 10:71

15. Kim JT, Jahan R, Saver JL, Investigators S (2016) Impact of glucose on outcomes in patients treated with mechanical thrombectomy: a post hoc analysis of the solitaire flow restoration with the intention for thrombectomy study. Stroke 47:120-127

16. Kunte H, Schmidt S, Eliasziw M, del Zoppo GJ, Simard JM, Masuhr F, Weih M, Dirnagl U (2007) Sulfonylureas improve outcome in patients with type 2 diabetes and acute ischemic stroke. Stroke 38:2526-2530

17. Mi D, Wang P, Yang B, Pu Y, Yang Z, Liu L (2018) Correlation of hyperglycemia with mortality after acute ischemic stroke. Ther Adv Neurol Disord 11:1756285617731686

18. Perez-Vega C, Domingo RA, Tripathi S, Ramos-Fresnedo A, Kashyap S, Quinones-Hinojosa A, Lin MP, Fox WC, Tawk RG (2021) Influence of glucose levels on clinical outcome after mechanical thrombectomy for large-vessel occlusion: a systematic review and meta-analysis. J Neurointerv Surg

19. Ribo M, Molina C, Montaner J, Rubiera M, Delgado-Mederos R, Arenillas JF, Quintana M, Alvarez-Sabin J (2005) Acute hyperglycemia state is associated with lower tPA-induced recanalization rates in stroke patients. Stroke 36:1705-1709 
20. Roman LS, Menon BK, Blasco J, Hernandez-Perez M, Davalos A, Majoie C, Campbell BCV, Guillemin F, Lingsma H, Anxionnat R, Epstein J, Saver JL, Marquering H, Wong JH, Lopes D, Reimann G, Desal H, Dippel DWJ, Coutts S, du Mesnil de Rochemont R, Yavagal D, Ferre JC, Roos Y, Liebeskind DS, Lenthall R, Molina C, Al Ajlan FS, Reddy V, Dowlatshahi D, Sourour NA, Oppenheim C, Mitha AP, Davis SM, Weimar C, van Oostenbrugge RJ, Cobo E, Kleinig TJ, Donnan GA, van der Lugt A, Demchuk AM, Berkhemer OA, Boers AMM, Ford GA, Muir KW, Brown BS, Jovin T, van Zwam WH, Mitchell PJ, Hill MD, White P, Bracard S, Goyal M, collaborators H (2018) Imaging features and safety and efficacy of endovascular stroke treatment: a meta-analysis of individual patient-level data. Lancet Neurol 17:895-904

21. Sang H, Li F, Yuan J, Liu S, Luo W, Wen C, Zhu Q, Chen W, Lin M, Qi L, Zhong Y, Wang Z, Ling W, Shi Z, Chen H, Liu W, Liu Z, Yao X, Xiong F, Zeng G, Hu X, Dong H, Mao A, Yang G, Huang J, Chen L, Gong Z, Tao J, Liu H, Wu D, Qiu Z, Yang Q, $\mathrm{Zi} \mathrm{W,} \mathrm{Li} \mathrm{F} \mathrm{(2021)} \mathrm{Values} \mathrm{of} \mathrm{baseline} \mathrm{posterior} \mathrm{circulation} \mathrm{acute}$ stroke prognosis early computed tomography score for treatment decision of acute basilar artery occlusion. Stroke 52:811-820

22. Saqqur M, Shuaib A, Alexandrov AV, Sebastian J, Khan K, Uchino K (2015) The correlation between admission blood glucose and intravenous rt-PA-induced arterial recanalization in acute ischemic stroke: a multi-centre TCD study. Int J Stroke 10:1087-1092

23. Schonewille WJ, Wijman CA, Michel P, Rueckert CM, Weimar C, Mattle HP, Engelter ST, Tanne D, Muir KW, Molina CA, Thijs V, Audebert H, Pfefferkorn T, Szabo K, Lindsberg PJ, de Freitas G, Kappelle LJ, Algra A, group Bs (2009) Treatment and outcomes of acute basilar artery occlusion in the Basilar Artery International Cooperation Study (BASICS): a prospective registry study. Lancet Neurol 8:724-730
24. Sheth KN, Kimberly WT, Elm JJ, Kent TA, Mandava P, Yoo AJ, Thomalla G, Campbell B, Donnan GA, Davis SM, Albers GW, Jacobson S, Simard JM, Stern BJ (2014) Pilot study of intravenous glyburide in patients with a large ischemic stroke. Stroke 45:281-283

25. Shi L, Rocha M, Leak RK, Zhao J, Bhatia TN, Mu H, Wei Z, Yu F, Weiner SL, Ma F, Jovin TG, Chen J (2018) A new era for stroke therapy: Integrating neurovascular protection with optimal reperfusion. J Cereb Blood Flow Metab. https://doi.org/10.1177/ 271678X18798162

26. Simard JM, Chen M, Tarasov KV, Bhatta S, Ivanova S, Melnitchenko L, Tsymbalyuk N, West GA, Gerzanich V (2006) Newly expressed SUR1-regulated NC(Ca-ATP) channel mediates cerebral edema after ischemic stroke. Nat Med 12:433-440

27. Turc G, Bhogal P, Fischer U, Khatri P, Lobotesis K, Mazighi M, Schellinger PD, Toni D, de Vries J, White P, Fiehler J (2019) european stroke organisation (ESO)- European society for minimally invasive neurological therapy (ESMINT) guidelines on mechanical thrombectomy in acute ischemic stroke. J Neurointerv Surg 11:535-538

28. Undas A, Wiek I, Stepien E, Zmudka K, Tracz W (2008) Hyperglycemia is associated with enhanced thrombin formation, platelet activation, and fibrin clot resistance to lysis in patients with acute coronary syndrome. Diabetes Care 31:1590-1595

29. Weyland CS, Mokli Y, Vey JA, Kieser M, Herweh C, Schonenberger S, Bendszuz M, Mohlenbruch MA, Ringleb PA, Nagel S (2021) Predictors for failure of early neurological improvement after successful thrombectomy in the anterior circulation. Stroke 52:1291-1298 